\title{
Erratum: A Toll-like receptor-independent antiviral response induced by double-stranded B-form DNA
}

Ken J Ishii, Cevayir Coban, Hiroki Kato, Ken Takahashi, Yuichi Torii, Fumihiko Takeshita, Holger Ludwig, Gerd Sutter, Koichi Suzuki, Hiroaki Hemmi, Shintaro Sato, Masahiro Yamamoto, Satoshi Uematsu, Taro Kawai, Osamu Takeuchi \& Shizuo Akira Nature Immunology 7, 40-48 (2005); published online 13 November 2005; corrected after print 10 March 2006

In the version of this article initially published, the GEO database accession number is missing. This should be the final subsection of Methods, as follows:

Accession code. GEO: microarray data, GSE4171.

The error has been corrected in the PDF version of the article.

\section{Erratum: A function for interleukin 2 in Foxp3-expressing regulatory T cells}

Jason D Fontenot, Jeffrey P Rasmussen, Marc A Gavin \& Alexander Y Rudensky

Nature Immunology 6, 1142-1151 (2005); published online 16 October 2005; corrected after print 10 March 2006

In the version of this article initially published, the GEO database accession number is missing. This should be the final subsection of Methods, as follows:

Accession code. GEO: microarray data, GSE4179.

The error has been corrected in the PDF version of the article. 